\title{
HEAVE MOTION OF A VERTICAL CYLINDER WITH HEAVE PLATES
}

\author{
Ewelina Ciba \\ Gdańsk University of Technology, Poland
}

\begin{abstract}
The shape of a vertical cylinder resembles the classic form of a spar platform. Spar platforms are floating platforms that are successfully used in waters of great depths and have several advantages that mean they are readily used in the oil industry. Many of these advantages are also relevant to their application for offshore wind turbines, which is currently being considered. In the hydrodynamic analysis of spar platforms, the determination of their hydrodynamic coefficients plays an important role. They can be determined based on the free decay test. The study presents a method for determining the hydrodynamic coefficients of an object based on the free decay test. The results of free oscillation calculations with the help of numerical fluid mechanics tools are presented and compared with the results of the experiment and analytical solution. The application of determined coefficients and their significance for floating platforms are discussed. The influence of change in the form of an additional damping element on the behaviour of spar structures is shown.
\end{abstract}

Keywords: spar platforms, heave plates, damping coefficient, added mass coefficient

\section{INTRODUCTION}

A spar is a type of floating platform designed for great depths. The structure of a spar platform is a vertical cylinder with positive buoyancy with an appropriate system of anchoring the platform to the seabed. They are always stable because their centre of buoyancy is above the centre of gravity. They can be used as mobile drilling rigs and their cable system (catenary) is easy to install, use and carry [1].

However, with long wave periods, the phenomenon of resonance may appear, which is largely influenced by damping related to water viscosity $[2,3]$. In [4], several alternative hull shapes that improve the damping in the vertical movement of the structure are presented. The best of the presented solutions is a heave plate, a damping plate attached to the floating structure that has a distinct advantage in reducing the motion response of a floating structure by increasing the added mass and damping.
This solution has become very popular and consistent results of analyses of its effectiveness can be found in the literature. The increase in the added mass, the damping coefficient and the related increase of the natural period under the influence of the heave plate have also been confirmed $[5,6,7]$.

Conventional offshore structure behavior calculations in offshore conditions are based on the potential theory and do not take into account viscous damping. This is a good approximation, but in some cases the viscosity should be included. Especially for the near resonance frequency [8]. The importance of viscosity also increases with the presence of edges in the structure, which was demonstrated by comparing the behavior of the hemispherical, rounded base and flat base structures in [9]

The resulting vortices and their impact on the drag coefficient depending on the geometry of the heave plate are shown e.g. in [10] or [11]. 
A good technique is to supplement linear theories with viscous drag coefficients obtained from experimental studies or calculations using the RANSE (Reynolds-Averaged Navier Stokes) model with turbulence models. This approach is described in [9] and [8]. The possibility to consider the effect of viscosity is also available in the commercial Ansys Aqwa software. Software uses three dimensional panel method to solve linearized hydrodynamic radioation and diffraction problem in frequency domain [12]

Potential flow solutions with added viscosity factors allow you to get good results in a reasonable amount of time. Timeconsuming RANSE calculations are performed only once to calculate the coefficients, and then a series of analyzes can be performed for different conditions and configurations with faster models based on the potential theory, extended by the coefficients mentioned.

In [13], modelling of the spar platform dynamics with the use of drag coefficient, inertia coefficient and added mass coefficient was presented. In addition, in [10], the added mass and damping coefficients related to the plate viscous damping were presented. One of the methods of estimating the viscous damping is to conduct free decay tests, as discussed in [7].

A very interesting and extensive analysis of the heaving plate impact using the CFD method is presented in [11] Detailed parametric studies were conducted numerically on spar with single and two heave plates. Effects of scaling ratio, diameter ratio, heave plate position to diameter of heave plate ratio, heave plate position to draft ratio, relative spacing and spacing between heave plates to draft ratio were examinated. As a result, the ranges of the analyzed parameters were given for which the heave motion reduction is the greatest.

In recent years, there has been a dynamic development of offshore wind energy. There are concepts of wind turbines based on various types of supporting structures. The concept of a turbine based on a jack-up platform is described in [14]. A concept based on a tension leg platform was presented in [15]. Most often, however, due to the large depths of the planned installation areas, the concepts of offshore wind turbines are based on spar-type platforms, as presented in
$[16,5]$. As a result, considerations regarding spar platforms are becoming very popular again. Due to the different nature of the forces acting in the case of offshore wind platforms than in the case of oil rigs, there are also new problems with the dynamics of these platforms related to the action of wind force.

This study presents the calculation results of the numerical fluid dynamics of the free decay test for a smooth cylinder and a cylinder with a heave plate (Fig. 1), and compares them with the results of analytical calculations and experiment.

The damping coefficients and the added mass coefficient are determined and compared with the values presented in the literature. The confirmation of the correctness of the applied method allows us to state that the computational fluid dynamics (CFD) calculations can be used to correctly estimate the hydrodynamic coefficients of the analysed platforms. This is of great importance in the process of designing a structure, as it allows to carry out extensive parametric analyses at a low cost and hence to choose the best solution, which should then be checked using model tests. Moreover, CFD methods allow for analyses of various shapes and configurations, which is not always possible with analytical methods.

\section{MATHEMATICAL DESCRIPTION OF THE PROBLEM}

Submerged, under the influence of the initial force, the cylinder will then make an oscillating motion relative to the equilibrium position, with the amplitude of the movement decreasing with time. A mathematical description of the problem can be found in the literature [17].

We assume that the moving body is a rigid body and we replace surface and volume forces by the movement of the point associated with the origin of the coordinate system. We also assume that the coefficients of the equation are constant over time, which causes the equation to become a linear. This assumption is only true for a small amplitude of motion. In this case, the deflection is not more than $6 \mathrm{~cm}$ so they can be used. The cylinder motion equation is given as:

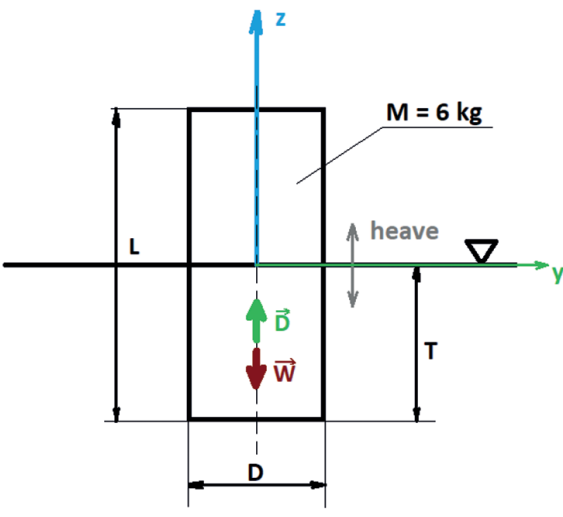

b)

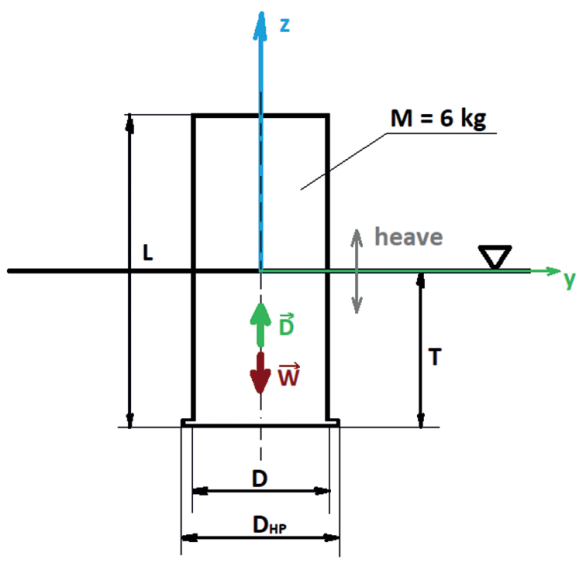

Fig. 1. Geometry of tested cylinders: a) smooth; $b$ ) with a heave plate 


$$
(m+a) \cdot \ddot{z}+b \cdot \dot{z}+c \cdot z=0
$$

where:

$\mathrm{z}$ - vertical displacement [m]

$\mathrm{m}-$ solid mass of cylinder $[\mathrm{kg}]$

a - hydrodynamic mass coefficient $[\mathrm{kg}]$

$\mathrm{b}$ - hydrodynamic damping coefficient $[\mathrm{kg} / \mathrm{s}]$

$c-$ restoring spring coefficient $\left[\mathrm{kg} / \mathrm{s}^{2}\right]$

Equation (1) can be written as:

$$
\ddot{z}+2 v \cdot \dot{z}+\omega_{0}^{2} \cdot z=0
$$

where the damping coefficient and the undamped natural frequency are defined as:

$$
2 v=\frac{b}{m+a} \quad \omega_{0}^{2}=\frac{c}{m+a}
$$

A non-dimensional damping coefficient $\kappa$ is defined as:

$$
\kappa=\frac{v}{\omega_{0}}=\frac{b}{2 \sqrt{(m+a) \cdot c}}
$$

By knowing the results of the free decay tests, we can calculate:

$$
\kappa=\frac{1}{2 \pi} \cdot \ln \left\{\frac{z_{a_{i}}-z_{a_{i+1}}}{z_{a_{i+2}}-z_{a_{i+3}}}\right\}
$$

Depending on the averaged displacement amplitude:

$$
\overline{z_{a}}=\left\{\frac{z_{a_{i}}-z_{a_{i+1}}}{z_{a_{i+2}}-z_{a_{i+3}}}\right\}
$$

We calculate the hydrodynamic added mass coefficient as:

$$
a=\frac{c}{\omega_{0}^{2}}-m
$$

In the case of initial displacement $z_{a}$, Eq. (2) takes the form:

$$
z=z_{a} e^{-v t}\left(\cos \omega_{z} t+\frac{v}{\omega_{z}} \sin \omega_{z} t\right)
$$

Due to the fact that for the frequency of free oscillations $\omega_{\mathrm{z}}{ }^{2}=\omega_{0}{ }^{2} \cdot v^{2}$ and damping is small $v<0.20, v^{2}<\omega^{2}$, we can skip $v^{2}$ and we can write that $\omega_{\mathrm{z}} \approx \omega_{0}$.

\section{RANSE CFD CALCULATIONS}

CFD calculations were made using STAR-CCM+. Unsteady calculations were performed in the three-dimensional domain, using the volume of fluid and K-epsilon turbulence models. Cylinder displacements were modelled using the dynamic fluid body interaction module using an overset mesh. Each of the boundary walls was given a no-slip wall condition. The computational domain was prepared as in Fig. 2.

The shape of the second cylinder was changed by a heave plate with diameter DHP $=0.178 \mathrm{~m}$ and thickness $\mathrm{t}=0.005 \mathrm{~m}$. The transition between the edge of the ring and the cylinder wall is rounded at radius $r=0.02 \mathrm{~m}$. The geometry of the cylinder with a heave plate is shown in Fig. 3.

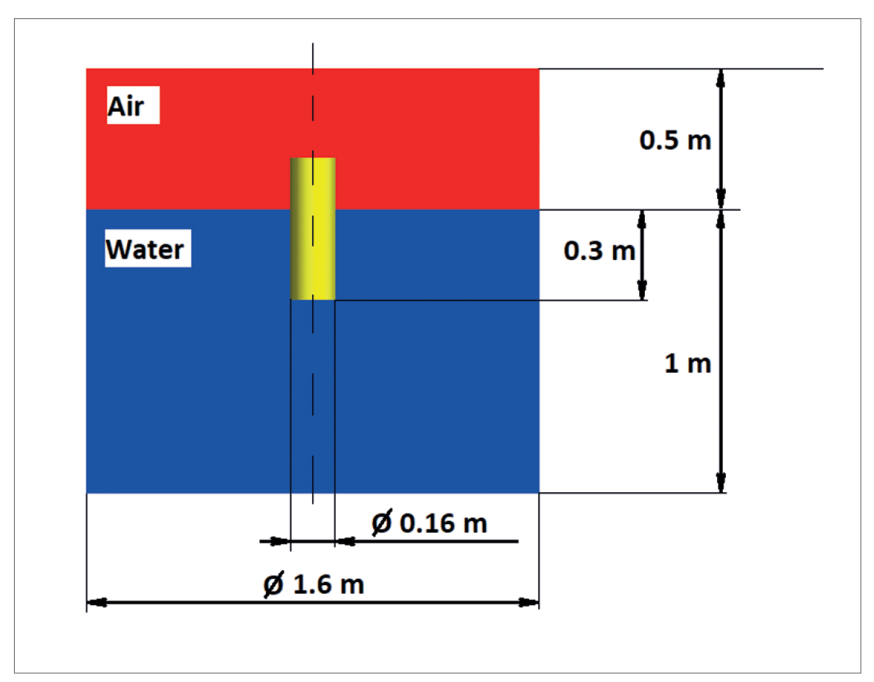

Fig. 2. Schematic of computational domain

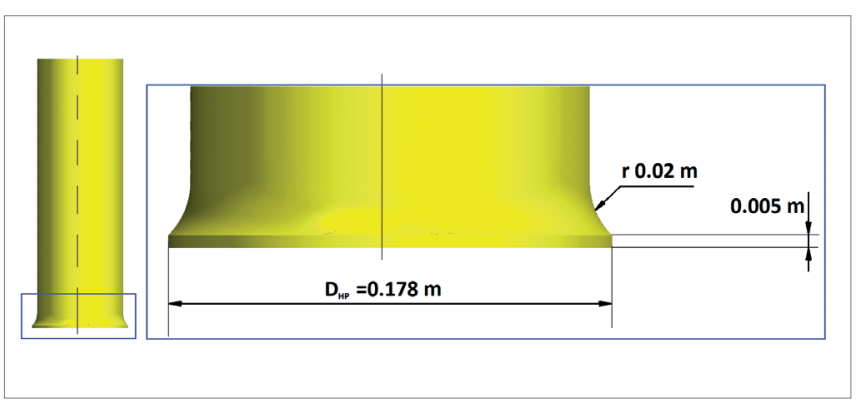

Fig. 3. Heave plate geometry

The assumed initial conditions were as follows: the share of water and air volume, and the initial pressure distribution of $1 \mathrm{~atm}$ above the free surface and hydrostatic pressure below the free surface. The cylinder was treated as a rigid body with one degree of freedom. The mass is equal to the displacement at draught $0.3 \mathrm{~m}$, i.e. $6 \mathrm{~kg}$. The initial solid velocity was assumed to force the initial cylinder displacment.

In the case of a smooth cylinder, the mesh was compacted around the cylinder $(0.005 \mathrm{~m})$ with a $0.002 \mathrm{~m}$ thick wall layer and two layers, by its expected displacement, and in the area of the free surface $(0.01 \mathrm{~m})$. The grid in the plane of symmetry with the division into moving and fixed parts is shown in Fig. 4 . The number of grid elements was over 6 million.

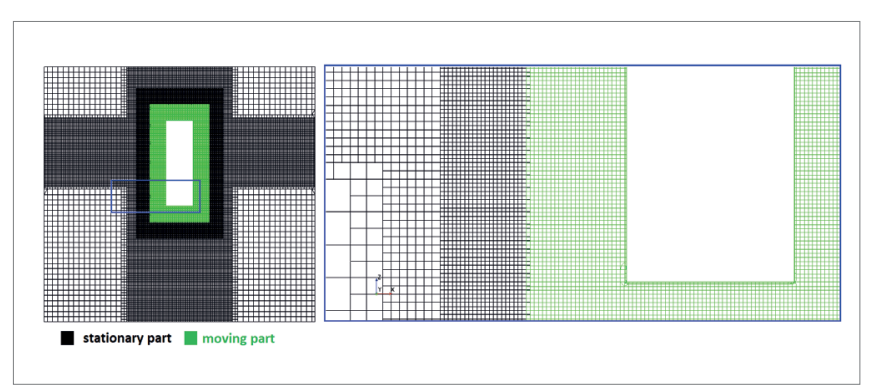

Fig. 4. Calculation grid in the plane of symmetry for smooth cylinder

In the case of a cylinder with an additional heave plate, the mesh was refinement in the plate area $(0.002 \mathrm{~m})$, the thickness of the prism layer was reduced to $0.0015 \mathrm{~m}$ and the number of layers was increased to seven. The mesh for the cylinder 
with the plate is shown in Fig. 5. The number of elements was increased to over 7 million.

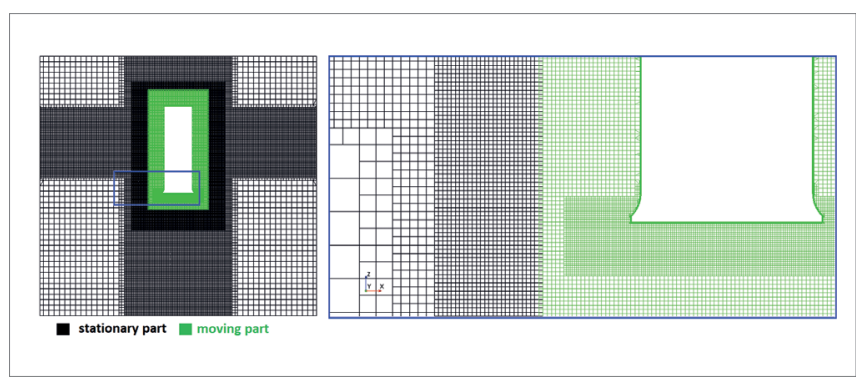

Fig. 5. Calculation grid for a cylinder with a heave plate

Calculations were performed for both cases with time step $\mathrm{t}_{\mathrm{k}}=0.01 \mathrm{~s}$.

\section{COMPARISON OF CALCULATION RESULTS WITH EXPERIMENT}

In order to validate the calculations, an experiment was conducted whose conditions were equivalent. The same mass and geometry were used, and the resulting draft of the structure. The oscillation was forced by the application of an external force, the effect of which then ceased. The force was selected in such a way that the first free displacement was the same as in the performed calculations. The experiment was carried out in such a way that the weight of the appropriate mass and the accelerometer were placed inside the empty cylinder of the set geometry. Then, an initial draft greater than the one resulting from the displacement balance was forced and released, recording the accelerations of the oscillating cylinder. It was assumed that the cylinder only moves vertically. Its slight deviations have been neglected. Two series of tests were performed for each case. A photograph from the study is shown in Fig. 6.

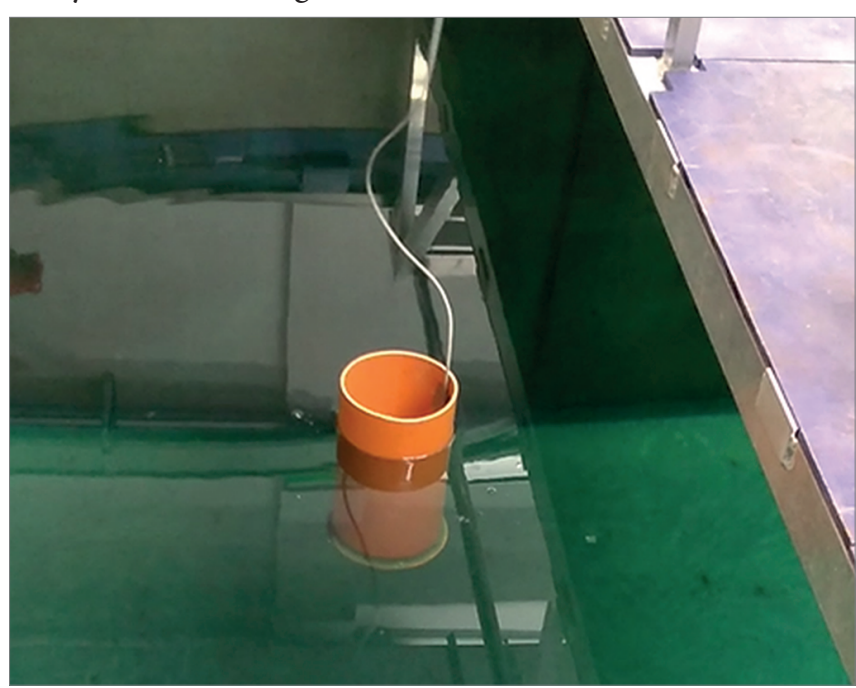

Fig. 6. Model tests of a damping ring cylinder

As a result of the measurements, the acceleration changes over time were obtained. The measured values for one of the test series are shown in Fig. 7.

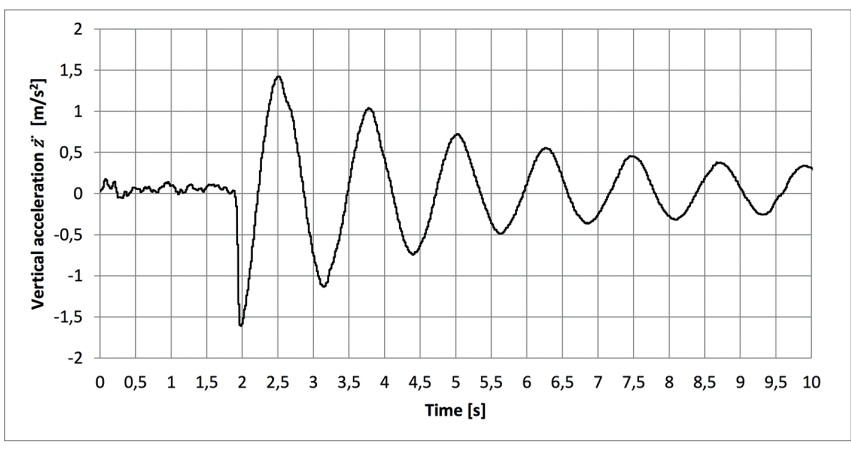

Fig. 7. Vertical cylinder acceleration measured for an example series of tests

Based on the measured accelerations, a displacement plot was created knowing that the amplitude is equal to the quotient of the acceleration and the square of the free vibration frequency at the points of maximum deflection.

$$
z_{a}=\frac{\ddot{z}}{\omega_{0}^{2}}
$$

The comparison of the displacement diagrams for both cases is shown in Fig. 8 for a smooth cylinder and in Fig. 9 for a cylinder with a heave plate.

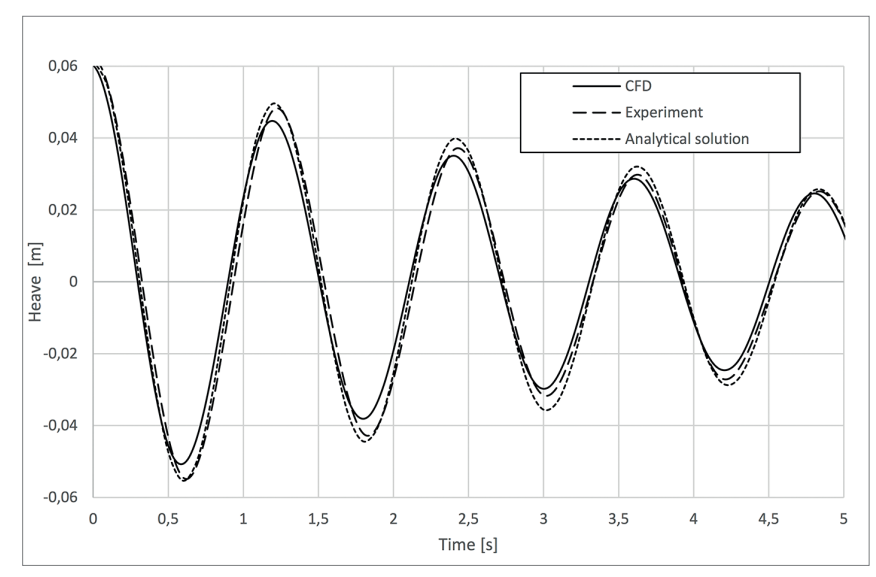

Fig. 8. Heaving a smooth cylinder

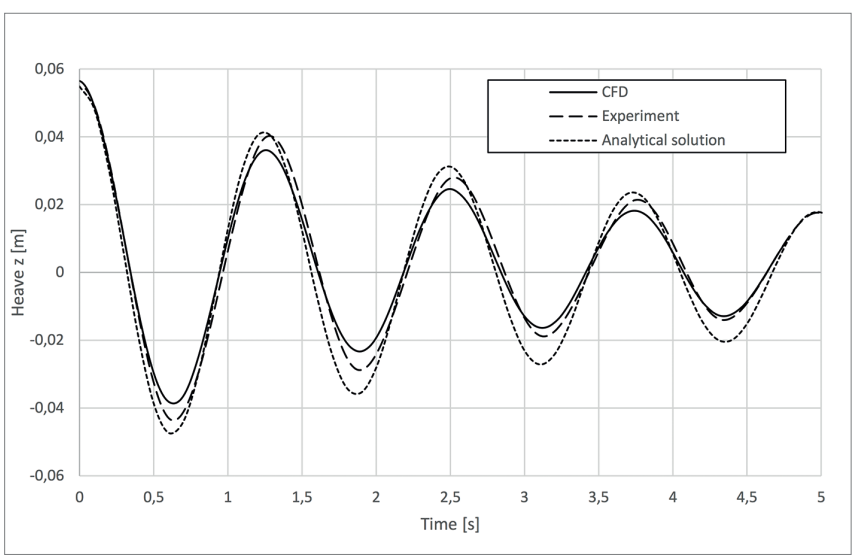

Fig. 9. Heaving a cylinder with a heave plate

To compare both cases, the non-dimensional damping coefficient $\kappa$ (Eq. (5)) is shown in the plot depending on the average amplitude $\overline{z_{a}}$ (Eq. (6)). 


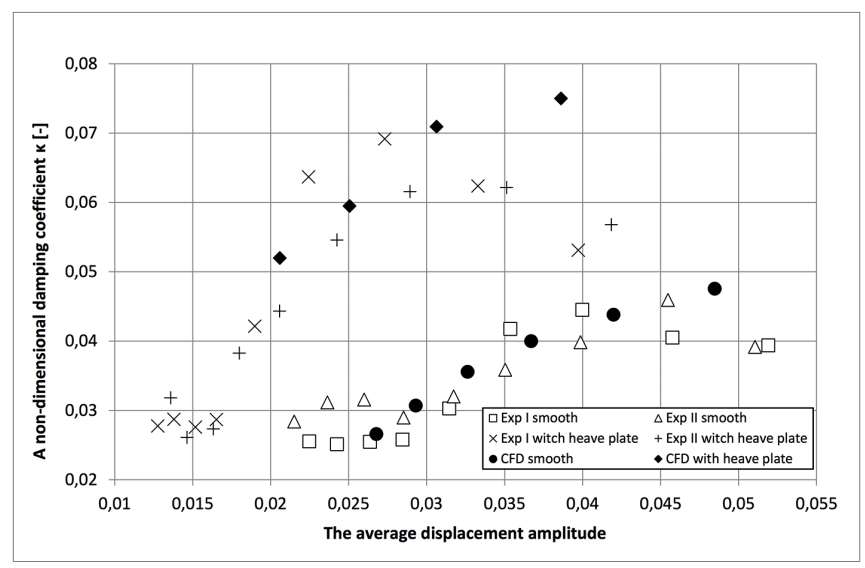

Fig. 10. A non-dimensional damping coefficient plot depending on the average displacement amplitude

The natural frequency $\omega_{0}$ (Fig. 11) and the added mass coefficient a (Fig. 12) are also compared.

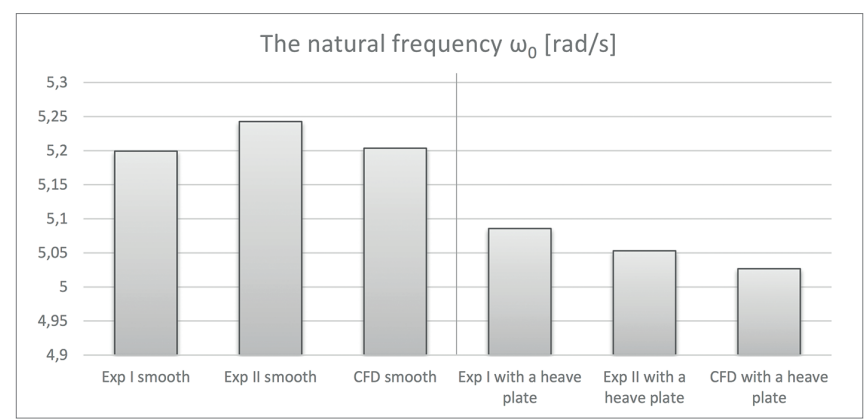

Fig. 11. Natural frequency

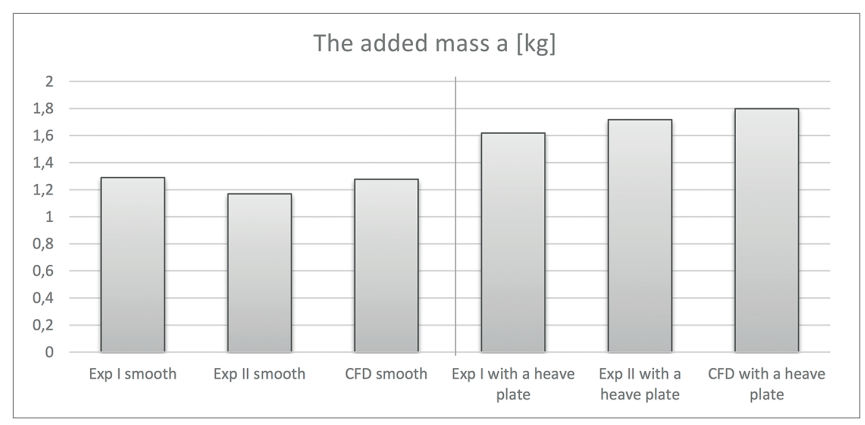

Fig. 12. Added mass coefficient

\section{SUMMARY}

The concepts of emerging wind platforms are often based on spar platforms and their modifications. This increases the interest in these platforms and the need for numerical analyses for the designed structures. Due to the time consuming nature of full RANSE CFD calculations, calculations by hybrid methods are a good solution, using much faster calculation methods based on hydrodynamic coefficients determined by RANSE CFD analyses and model tests. This study presents a method for determining such coefficients. Based on the results presented, it can be concluded that CFD calculations allow us to reliably map reality. In both cases, the large convergence of CFD calculation results, analytical solutions and experiment results was obtained, and the differences in the dimensionless attenuation coefficient are not greater than the differences between the values obtained from both series of the experiment (fig. 11 and 12). The results of the calculations and the experiment differ for greater displacement amplitudes. Perhaps the calculations should be repeated with a shorter time step to improve convergence for these points.

As shown in the diagram (Fig. 10), the value of the damping coefficient depends on the displacement amplitude, and therefore the cylinder velocity. The conclusion is that when extending the calculations based on the potential method with coefficients related to viscosity, either the average damping coefficient for the anticipated speed range should be used or, if possible, the value of the coefficient as a function of velocity should be used.

Comparing the results for the cases of a smooth cylinder and a cylinder with an additional heave plate, even a slight change in shape whilst maintaining the same weight of the structure causes an increase in damping. An additional element also reduces the frequency of oscillations and causes an increase in the mass of the accompanying water. The obtained values of the coefficients are similar to those obtained by other methods presented in the literature. A further conclusion can be drawn from this that when designing the geometry of a spar platform, it is worth considering the use of additional damping elements of this type.

Confirmation of the correct application of CFD methods for the analysis of hydrodynamic coefficients is of great importance for the analysis of various concepts of additional damping elements for structures for offshore wind turbines. This will allow, at relatively low costs, for the analysis of many different solutions and we can select the best of them for model tests necessary to verify the obtained results. It is can be used to carry out calculations for plates with jagged edges, equipped with movable elements, and for flexible plates. CDF analyses allow us to carry out many series of calculations depending on changes in individual parameters of the elements (e.g. increasing the diameter of the plate) that will also help to trace the dependence of the coefficients on the selected parameters.

\section{ACKNOWLEDGEMENT}

The calculations were performed using a Simcenter STARCCM+ license.

\section{REFERENCES}

1. Jain A.K., Agarwal A.K. (2003): Dynamic Analysis of Offshore Spar Platforms. Defence Science Journal, Vol. 53, No. 2, pp. 211-219

2. Sharman K. T., Robertson A., Lewis J. (2019): Heave Plate Hydrodynamics for Offshore Wind Turbine Applications. EERA DeepWind, Trondheim 
3. Liu Y. Yan H. Yung T. W. (2010): Nonlinear Resonant Response of Deep Draft Platforms in Surface Waves. ASME 2010 29th International Conference on Ocean, Offshore and Arctic Engineering

4. Bhatta D. D. (2007): Computation of Added Mass and Damping Coefficients due to a Heaving Cylinder. J. Appl. Math. Comput., 23, 127140

5. Gilloteaux J. Ch., Bozonnet P. (2014): Parametric Analysis of a Cylinder Like Shape Floating Platform Dedicated to MultiMega Watt Wind Turbine. IFP Energies Nouvelles, Applied Mechanics division Rueil-Malmaison, France.

6. Fisher F. J., Gopalkrishnan R. (1998): Some Observations on the Heave Behavior of Spar Platforms. Journal of Offshore Mechanics and Arctic Engineering.

7. Koh H. J., Cho I. J. (2016): Heave Motion Response of a Circular Cylinder with the Dual Damping Plates. Ocean Engineering, 125, 95-102.

8. Zhao Ch., Cao F., Shi H. (2020): Optimisation of heaving buoy wave energy converter using a combined numerical model Applied Ocean Research 102 (2020) 102208

9. Gu, H., Stansby, P., Stallard, T., \& Carpintero Moreno, E. (2018). Drag, added mass and radiation damping ofoscillating vertical cylindrical bodies in heave and surge in still water. JOURNAL OF FLUIDS AND STRUCTURES, 82, 343-356. https://doi.org/10.1016/j.jfluidstructs.2018.06.012

10. Tao, L., Cai, S. (2004): Heave Motion Suppression of a Spar with a Heave Plate. Ocean Engineering.

11. A. Subbulakshmi, R. Sundaravadivelu (2016) Heave damping of spar platform for offshore wind turbine with heave plate Ocean Engineering 121 (2016) 24-36

12. S. Sudhakar, S. Nallayarasu (2011) Influence of Heave Plate on Hydrodynamic Response of Spar OMAE2011-49565

13. Haslum, H. A. (2000): Simplified Methods Applied to Nonlinear Motion of Spar Platforms. Norwegian University of Science and Technology, Trondheim.

14. Dymarski P. (2019): Design of Jack-up Platform for 6 MW Wind Turbine: Parametric Analysis Based Dimensioning of Platform Legs. Polish Maritime Research, 26, 183-197.

15. Żywicki J., Dymarski P., Ciba E., Dymarski C. (2017): Design of Structure of Tension Leg Platform for 6 MW Offshore Wind Turbine Based On Fem Analysis. Polish Maritime Research, Vol.-24, s1, 230-241

16. Dymarski P., Dymarski C., Ciba E. (2019): Stability Analysis of the Floating Offshore Wind Turbine Support Structure of Cell Spar Type During its Installation. Polish Maritime Research, Vol. 26, 4(104), 109-116.

17. Journee J. M. J., Massie W. W. (2001): Offshore Hydromechanics. Delft University of Technology.

18. Kim et al. M.-H. (2012): Spar platforms: Technology and Analysis Methods. American Society of Civil Engineers.

\section{CONTACT WITH THE AUTHOR}

\author{
Ewelina Ciba \\ e-mail: ewelina.ciba@pg.edu.pl \\ Gdańsk University of Technology \\ Narutowicza 11/12 \\ 80-233 Gdańsk \\ Poland
}

